\title{
Distinct displacements of the optic radiation based on tumor location revealed using preoperative diffusion tensor imaging
}

\author{
Katharina Faust, MD, and Peter Vajkoczy, MD \\ Department of Neurosurgery, Charité University Hospital, Berlin, Germany
}

OBJECTIVE Visual field defects (VFDs) due to optic radiation (OR) injury are a common complication of temporal lobe surgery. The authors analyzed whether preoperative visualization of the optic tract would reduce this complication by influencing the surgeon's decisions about surgical approaches. The authors also determined whether white matter shifts caused by temporal lobe tumors would follow predetermined patterns based on the tumor's topography.

METHODS One hundred thirteen patients with intraaxial tumors of the temporal lobe underwent preoperative diffusion tensor imaging (DTI) fiber tracking. In 54 of those patients, both pre- and postoperative VFDs were documented using computerized perimetry. Brainlab's iPlan 2.5 navigation software was used for tumor reconstruction and fiber visualization after the fusion of DTI studies with their respective magnetization-prepared rapid gradient-echo (MP-RAGE) images. The tracking algorithm was as follows: minimum fiber length $100 \mathrm{~mm}$, fractional anisotropy threshold 0.1. The lateral geniculate body and the calcarine cortex were employed as tract seeding points. Shifts of the OR caused by tumor were visualized in comparison with the fiber tracking of the patient's healthy hemisphere.

RESULTS Temporal tumors produced a dislocation of the OR but no apparent fiber destruction. The shift of white matter tracts followed fixed patterns dependent on tumor location: Temporolateral tumors resulted in a medial fiber shift, and thus a lateral transcortical approach is recommended. Temporopolar tumors led to a posterior shift, always including Meyer's loop; therefore, a pterional transcortical approach is recommended. Temporomesial tumors produced a lateral and superior shift; thus, a transsylvian-transcisternal approach will result in maximum sparing of the fibers. Temporocentric tumors also induced a lateral fiber shift. For those tumors, a transsylvian-transopercular approach is recommended. Tumors of the fusiform gyrus generated a superior (and lateral) shift; consequently, a subtemporal approach is recommended to avoid white matter injury. In applying the approaches recommended above, new or worsened VFDs occurred in $4 \%$ of the patient cohort. Total neurological and surgical morbidity were less than $10 \%$. In $90 \%$ of patients, gross-total resection was accomplished.

CONCLUSIONS Preoperative visualization of the OR may help in avoiding postoperative VFDs.

http://thejns.org/doi/abs/10.3171/2015.3.JNS141584

KEY WORDS DTI; fiber tracking; temporal lobe; tumor surgery; visual field defect; optic radiation; oncology

$\mathrm{N}$ EW visual field defects (VFDs) through optic radiation (OR) injury constitute the most common complication following temporal lobe surgery. The frequency of VFDs after temporal lobe surgery is reported to be as high as $20 \%-100 \%$ in previous studies. ${ }^{10,12,30}$ Postoperative VFDs after anterior temporal lobe resection, as conducted in epilepsy surgery, for example, have been estimated around $70 \%$, depending on the extent of resection. ${ }^{24}$ This enormous visual morbidity is easily explained by the anatomy of the human OR, which traverses a major expanse of the temporal lobe. Of all the white matter tracts within the temporal lobe network, the OR is the largest. ${ }^{32}$

Recent technical progress has enabled the visualization of fiber tracts in the living subject through diffusion tensor imaging (DTI). Before that, knowledge of the visual pathway was based mainly on fiber dissection studies in deceased subjects ${ }^{6,15,21}$ and partly on the analysis of postoperative visual field deficits. ${ }^{9,13,28}$ The underlying physics of the DTI technique have been described well and extensively, ${ }^{1,2,5,14,17}$ as have the technique's physical limitations..$^{25}$

ABBREVIATIONS CP = computerized perimetry; DTI = diffusion tensor imaging; GBM = glioblastoma multiforme; LGB = lateral geniculate body; MP-RAGE = magnetization-prepared rapid gradient-echo; OR = optic radiation; VFD = visual field defect; $\mathrm{VOI}=$ volume of interest.

SUBMITTED July 6, 2014. ACCEPTED March 12, 2015.

INCLUDE WHEN CITING Published online October 2, 2015; DOI: 10.3171/2015.3.JNS141584. 
A variety of different tracking algorithms have been employed. ${ }^{20}$

Clearly, the visualization of the anterior visual pathway using DTI is difficult, with the proximity of air-filled spaces in the bony structures of the anterior and middle cranial fossae, as well as the many crossing fibers and small fiber diameters, leading to artifacts..$^{22}$ However, the posterior portion of the visual tracts from the lateral geniculate body (LGB) onward have been clearly and reproducibly illustrated, despite varying tracking algorithms..$^{20,23,31}$

To date, the majority of tractography studies of the visual pathway have been undertaken in healthy subjects, mainly in the context of anatomical investigations. ${ }^{9,21,23,34}$ Here, we aimed to analyze whether DTI would prove feasible in the diseased brain of patients with space-occupying lesions. After all, it can be assumed that infiltrative tumors growing in the direction of the OR can lead to destruction of the fibers or at least interference with their visualization by DTI. Alternatively, it is conceivable that fibers stay largely intact and are merely shifted in another direction by the tumor's mass effect. This finding would substantiate the suitability of fiber tracking for neurosurgical planning in temporal lobe tumor surgery. We then tested the hypothesis that the supposed fiber shifts follow predetermined patterns based on the tumor's topography.

Recently, we established a classification of temporal lobe tumors and suggested the respective surgical approaches; ${ }^{7}$ however, these approaches were based solely on anatomical considerations. In that tumor series we found a considerably low incidence of new postoperative VFDs. We, therefore, hypothesized that the suggested surgical approaches largely respect the fiber integrity of the OR. This prompted us to further investigate this finding by expanding our series of temporal lobe tumors and incorporating preoperative DTI fiber tracking to visualize the relation of these tumors to the OR.

Hence the second aim of our study was to determine whether preoperative visualization of the optic tract would reduce the complication of postoperative VFDs by influencing a surgeon's decisions about surgical approaches.

\section{Methods \\ Patients}

One hundred thirteen patients with intraaxial tumors of the temporal lobe, who had undergone preoperative DTI fiber tracking, were included in this study. Thirty patients from our previously published cohort, ${ }^{7}$ who had adequate DTI data sets with their preoperative imaging, were also included in this study cohort. The inclusion criteria were as follows: 1) tumor confined to the temporal lobe with anatomical vicinity to the OR; 2) intraaxial, infiltrative growing tumor; and 3) preoperative DTI sequence, as well as pre- and postoperative evaluations of visual fields. The exclusion criteria were as follows: 1) tumor transgressing the frontal, parietal, or occipital lobe; 2) extraaxial tumor (for example, meningioma) or vascular lesion; 3) previous temporal lobe injury (for example, recurrent tumor) or occipital lobe injury; 4) pacemaker or other circumstance precluding MRI; and 5) relevant preexisting visual im- pairment due to disease of the anterior optic pathway (for example, ocular blindness).

All patients had received high-dose glucocorticoids to reduce peritumoral edema for several days preoperatively and before perimetry.

\section{Magnetic Resonance Imaging Data Acquisition}

Diffusion tensor imaging data sets were acquired using an 8-channel head coil and a 1.5-T MRI unit (GE Healthcare). The tensors were averaged by repeating a diffusionweighted single-shot echo planar sequence along 23 different geometric directions with a slice thickness of $3 \mathrm{~mm}$, b-value of $1000 \mathrm{sec} / \mathrm{mm}^{2}$, TR of $11.000 \mathrm{msec}$, TE of 83 msec, matrix size of $128 \times 128$, and FOV of $240 \times 240$ $\mathrm{mm}$. A total of 1008 images were generated. Data acquisition time was approximately 8 minutes.

In addition, conventional MRI was performed in the same session, including a 1-mm slice thickness T1 inversion-recovery 3D gradient-echo (magnetization-prepared rapid gradient-echo [MP-RAGE]) sequence among other conventional sequences commonly employed for tumor imaging.

\section{Diffusion Tensor Imaging Processing and Fiber Tracking}

Brainlab's iPlan 2.5 navigation software was used for tumor reconstruction and fiber visualization after fusion of the DT images with their respective MP-RAGE images. The MP-RAGE and DTI files were converted into a single multivolume file and then corrected for eddy currents and subject movements using the iPlan "eddy-correction" algorithm. The DTI data sets were fused to the MPRAGE data sets using the "autofuse" algorithm of the iPlan 2.5. The seed point regions for tractography were based on anatomical landmarks used in previously published work by other groups. ${ }^{4,18,20,33}$ Rectangular volumes of interest (VOIs) were defined in the coregistered anatomical data sets (T1-MPRAGE and T2). Two seed points were chosen: the LGB (VOI-1) and the calcarine cortex (VOI-2). The LGB was identified on axial sections at the level of transition from the posterior limb of the internal capsule to the cerebral peduncle, posterolateral to the peduncle. ${ }^{18}$ The VOI-2 was allocated to Brodmann area 19. Fiber tracts passing through both VOIs were considered to compose the OR. Stray fibers extending to the contralateral hemisphere or the frontal lobe were removed using the iPlan 2.5 "exclude" algorithm. A fractional anisotropy threshold of 0.1 and a minimum fiber length of $100 \mathrm{~mm}$ were applied as standard settings for all trackings based on tracking algorithms for the OR, as previously used by other groups. ${ }^{29,33,34}$ Shifts of the OR caused by the tumor were visualized in comparison with the fiber tracking of the patient's healthy hemisphere. To gain supplementary quantitative data on tract volumes and numbers of fibers, fiber objects were created using Brainlab's "create 3D object" function. Using the function "plan content," the volume of the fiber objects as well as the number of fibers within the chosen fiber object was given. The mean OR volumes and mean number of fibers were compared between the tumor-bearing hemisphere and the healthy hemisphere of each patient. 


\section{Integration Into Surgical Planning}

Tractography results and reconstructions of the shifted OR were preoperatively presented to the surgeons, who were given the opportunity to consider the data and adjust their surgical approach accordingly. Based on emerging patterns of fiber shifts, tumor locations were assigned to optimum surgical approaches aimed at the best possible sparing of fibers. Postoperative neurological and surgical outcomes were registered.

\section{Evaluation of Visual Field Deficits}

Both pre- and postoperative VFDs were compared using computerized perimetry (CP) and finger perimetry. Of the 113 patients included in the study, 54 underwent preoperative and postoperative $\mathrm{CP}$ by a board-certified ophthalmologist, whereas 59 patients underwent preand postoperative finger perimetry for the evaluation of VFDs. Since the computer-based record of VFDs has better accuracy and must therefore be considered a higher standard, only the 54 patients with CP were statistically analyzed.

All preoperative $\mathrm{CP}$ studies were performed within 48 hours before surgery after the patient had already received a course of corticosteroids for several days. All postoperative $\mathrm{CP}$ studies were performed between postoperative Days 3 and 6 (usually on the day of discharge), when the immediate postoperative swelling was assumed to have partially subsided. Again, all patients were still on corticoids during postoperative $\mathrm{CP}$. All $\mathrm{CP}$ studies were performed at the same institution.

\section{Results}

Tumor pathologies are summarized in Table 1. In all 113 patients, the optic tract could be visualized by the chosen tracking algorithm in both hemispheres, regardless of the tumor's composition, grade, size, or location within the temporal lobe. In none of the cases were fibers pervading the tumor, and neither was there apparent fiber loss or thinning. Even for very large lesions, no distinguishable loss of fibers was noticed (Fig. 1). The mean OR volume in the tumor-bearing hemisphere was $13.59 \pm 2.77 \mathrm{~cm}^{3}$, as compared with $14.61 \pm 2.20 \mathrm{~cm}^{3}$ in the healthy hemisphere. Thus, the tract volumes were slightly greater in the healthy hemisphere; however, the 2-tailed $\mathrm{p}$ value was

TABLE 1. Characteristics of 113 patients who underwent temporal lobe surgery for intraaxial tumors

\begin{tabular}{lc}
\hline \multicolumn{1}{c}{ Characteristic } & No. \\
\hline Mean patient age in yrs & $54 \pm 16$ \\
\hline M/F ratio & $70: 43$ \\
\hline Tumor pathology & \\
\hline GBM & 65 \\
\hline Astrocytoma GIII & 18 \\
\hline Astrocytoma GII & 10 \\
\hline Oligodendroglioma GII & 3 \\
\hline Metastasis & 17 \\
\hline
\end{tabular}

GII, GIII = Grade I, Grade III; GBM = glioblastoma multiforme.
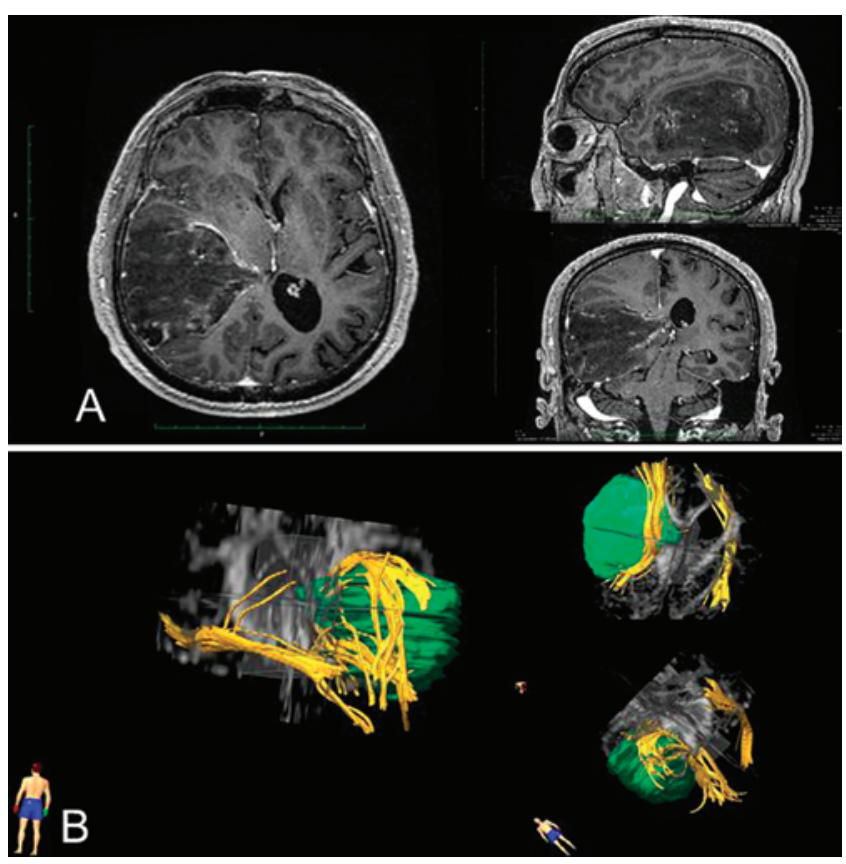

FIG. 1. A: Images depicting a very large temporolateral tumor (oligodendroglioma) occupying a great extent of the temporal lobe. B: Tractography revealing maximum medial displacement of the OR (yellow) by tumor (green), which eventually led to a splitting of the OR into a superior and an inferior bundle. There is no apparent destruction of the $\mathrm{OR}$ by tumor, even though the lesion is very large. Figure is available in color online only.

0.058 , which is considered not very significant. The slight difference may be explained by compression of the tracts by tumor (Fig. 2).

The number of OR fibers in the tumor-bearing hemisphere was $248 \pm 57.5$ as compared with $257 \pm 71.5$ in the healthy hemisphere. The $\mathrm{p}$ value was 0.721 , indicating no significant difference (Fig. 3).

Likewise, in invasively growing tumors like glioblastoma multiforme (GBM) or lower grade astrocytoma, no fibers within the tumor mass were detected using the cho-

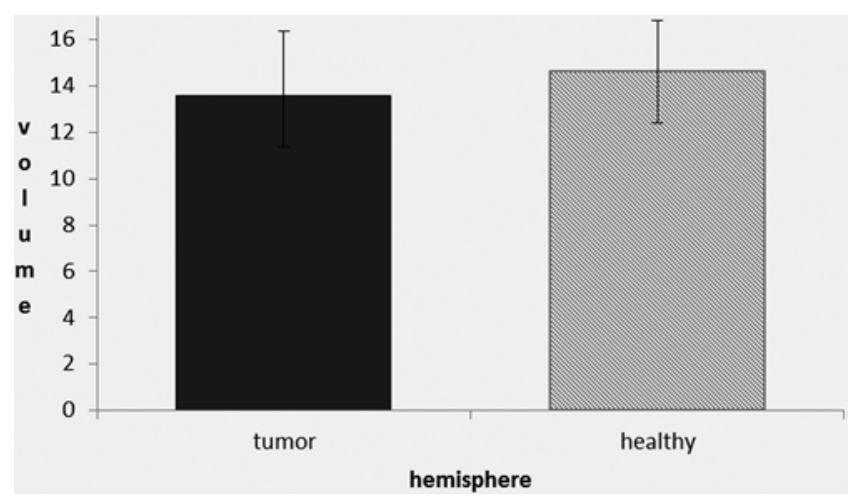

FIG. 2. Volumetry of the OR comparing the tumor-affected hemisphere with the healthy hemisphere. Values on the $y$-axis are expressed as the means \pm standard deviations in $\mathrm{cm}^{3}$. No significant difference was seen $\left(13.59 \pm 2.77 \mathrm{~cm}^{3}\right.$ vs $\left.14.61 \pm 2.20 \mathrm{~cm}^{3}, p=0.058\right)$. Figure is available in color online only. 


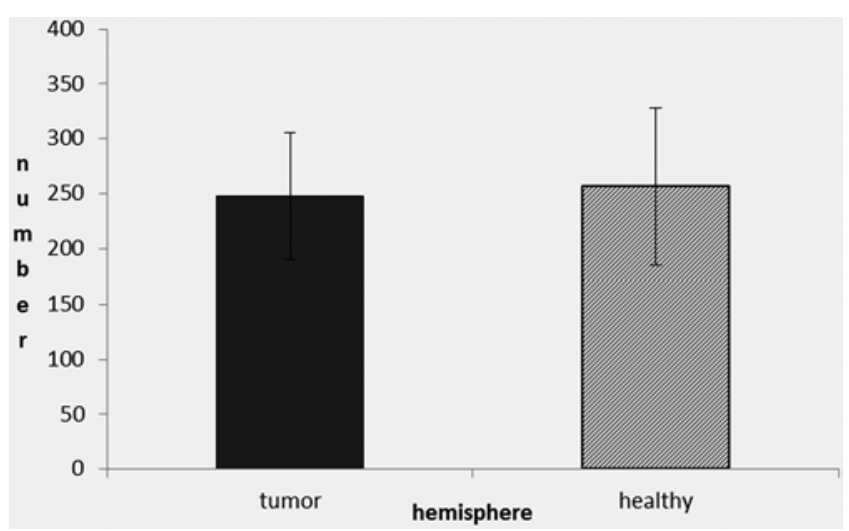

FIG. 3. Fiber count of the OR comparing the tumor-affected hemisphere to the healthy hemisphere. Values on the y-axis are expressed as the mean \pm standard deviation number of fibers. No significant difference was seen ( $248 \pm 57.5$ vs $257 \pm 71.5, p=0.721)$. Figure is available in color online only.

sen algorithm. No difference was noticed in the behavior of fiber shifts between rather sharply bordered tumors, like metastases, and diffusely infiltrating tumors, like GBM. In all cases, clear displacement of the fiber tracts, without apparent injury to the fibers, was observed in proximity to the tumor. The direction of fiber displacement depended on the anatomical tumor location.

\section{Patterns of Fiber Shifts}

To further analyze possible fiber shift patterns that depended on the anatomical tumor location, we divided the temporal lobe into 4 main regions, based on a previously described anatomical compartmentation of the temporal lobe. $^{7}$

\section{Temporolateral Tumors}

Lateral tumors were located in the inferior, middle, or superior temporal gyrus. Thirty-five tumors were lateral. In all of these cases, the OR was shifted medially (Fig. 4). Thus, a lateral transcortical approach to the tumor will not injure any of the fiber tracts.

\section{Temporopolar Tumors}

The temporopolar region constitutes the most anterior part of the temporal lobe, always including Brodmann area 38. The 8 temporopolar tumors in this study always caused posterior buckling of the OR (Fig. 5). Even though Meyer's loop often could not be clearly distinguished as a separate bundle, we must assume that the anterior bundle was subject to the strongest buckling. An anterior approach by a pterional craniotomy will not transect any of the OR fibers.

\section{Temporomesial Tumors}

Temporomesial tumors were confined to the hippocampus, parahippocampus, amygdala, and uncus. Those limbic tumors grow from the most medial region of the temporal lobe outwards. For each of the 36 temporomesial tumors included in this study, the fiber tracts shifted laterally (and often cranially). In none of the mesial lesions were fibers shifted medially or inferiorly (Figs. 6 and 7). Consequently, lateral transcortical approaches to the temporomesial region carry a high risk of transecting optic fibers. A medial approach, that is, transsylvian, does not interfere with the course of the OR.

\section{Temporocentric Tumors}

Even with tumors of the temporal stem, which are located in the midst of the comprehensive white matter network in the temporal lobe, visualization of the OR was

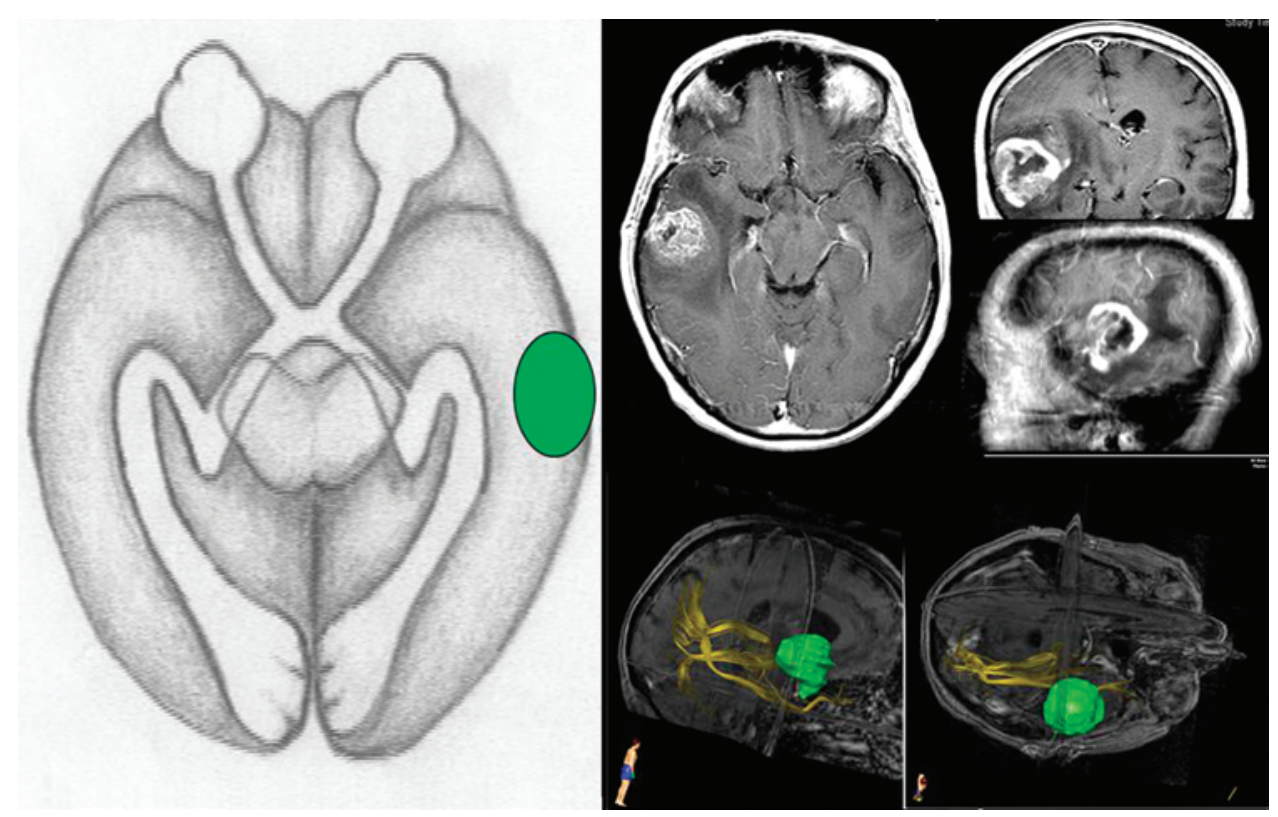

FIG. 4. Example of a temporolateral tumor. As depicted, fiber tracts of the OR (yellow) are shifted medially by the tumor (green). Figure is available in color online only. 


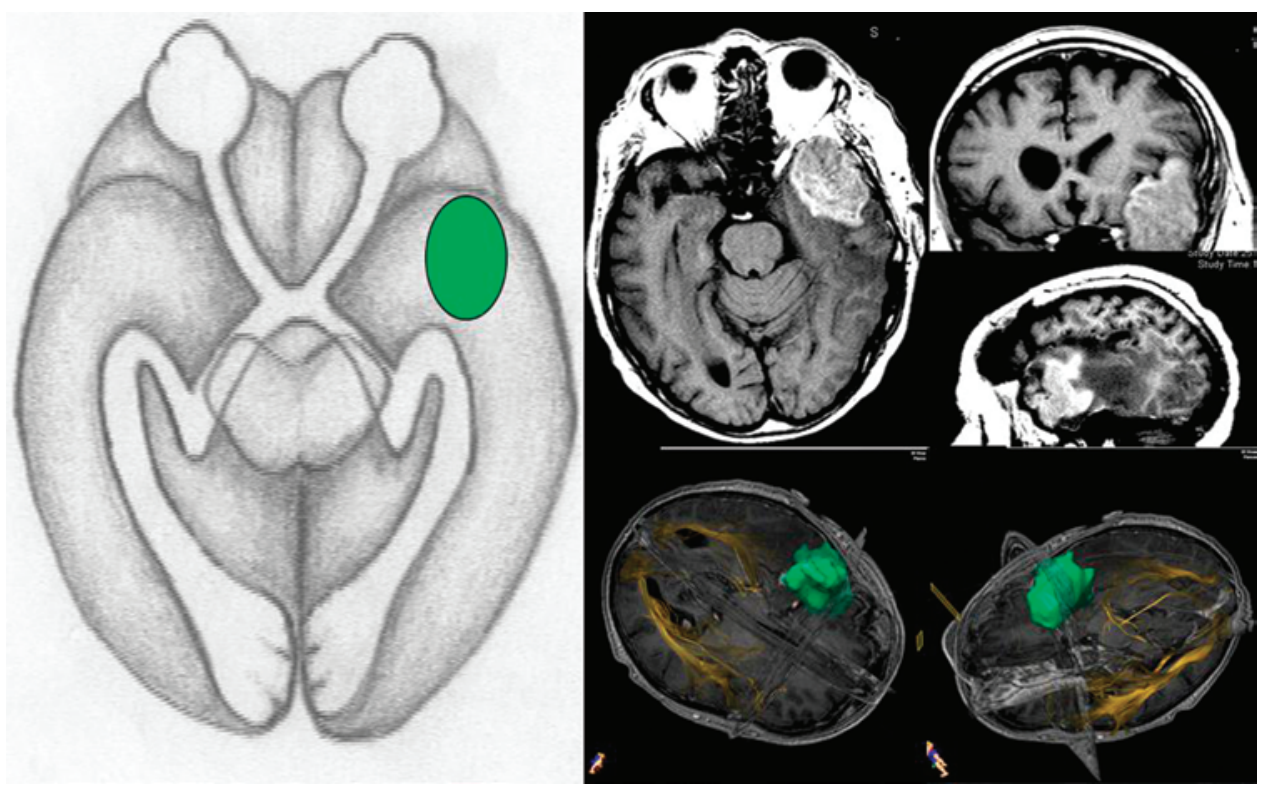

FIG. 5. Example of temporopolar tumor. As depicted, fiber tracts of the OR (yellow) are shifted dorsally by the tumor (green). Figure is available in color online only.

clearly feasible in every case. Each of the 32 temporocentric tumors in this study shifted the optic fibers laterally and superiorly. Again, for none of the temporocentric tumors did we observe a mesial or inferior shift (Fig. 8).

\section{Fusiform Gyrus Tumors}

Tumors of the medial and lateral occipitotemporal (fusiform) gyri abut the temporal base. These tumors cause a superior and slightly lateral shift of the optic tract. Hence, a lateral transcortical approach to the basal gyri will transect the optic fibers. The shortest trajectory to the tumor through a subtemporal approach bears no risk of direct injury. However, only 2 temporobasal tumors were in this cohort.

Altogether, there was a high correlation between tumor location and the direction of OR fiber shift. In all cases, the OR fiber shifts followed the above-described unequivocal patterns. Thus, standard surgical approaches could be ascribed to the tumor locations with the aim of minimizing injury to the OR (Fig. 9).

\section{Surgical Outcome}

Before operations, the surgeons received the results of
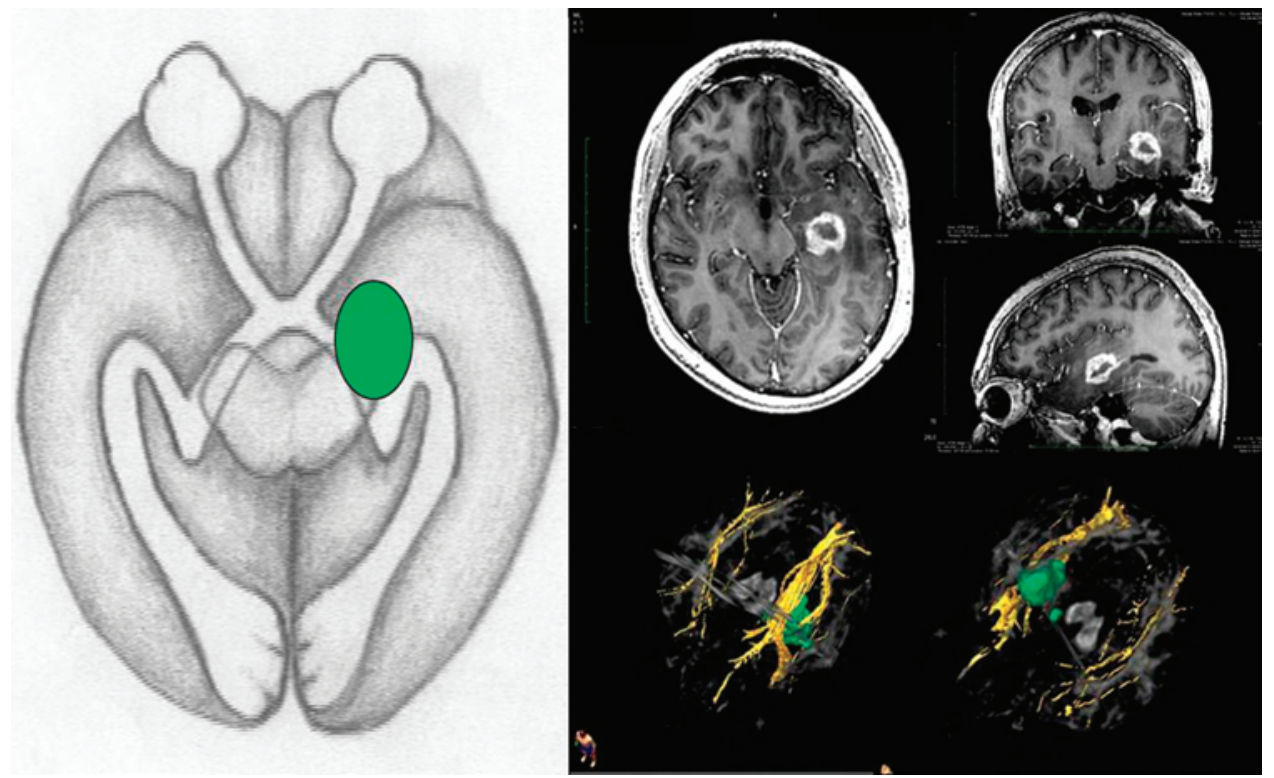

FIG. 6. Example of temporomesial tumor. As depicted, fiber tracts of the OR (yellow) are shifted laterally and superiorly by the tumor (green). Figure is available in color online only. 


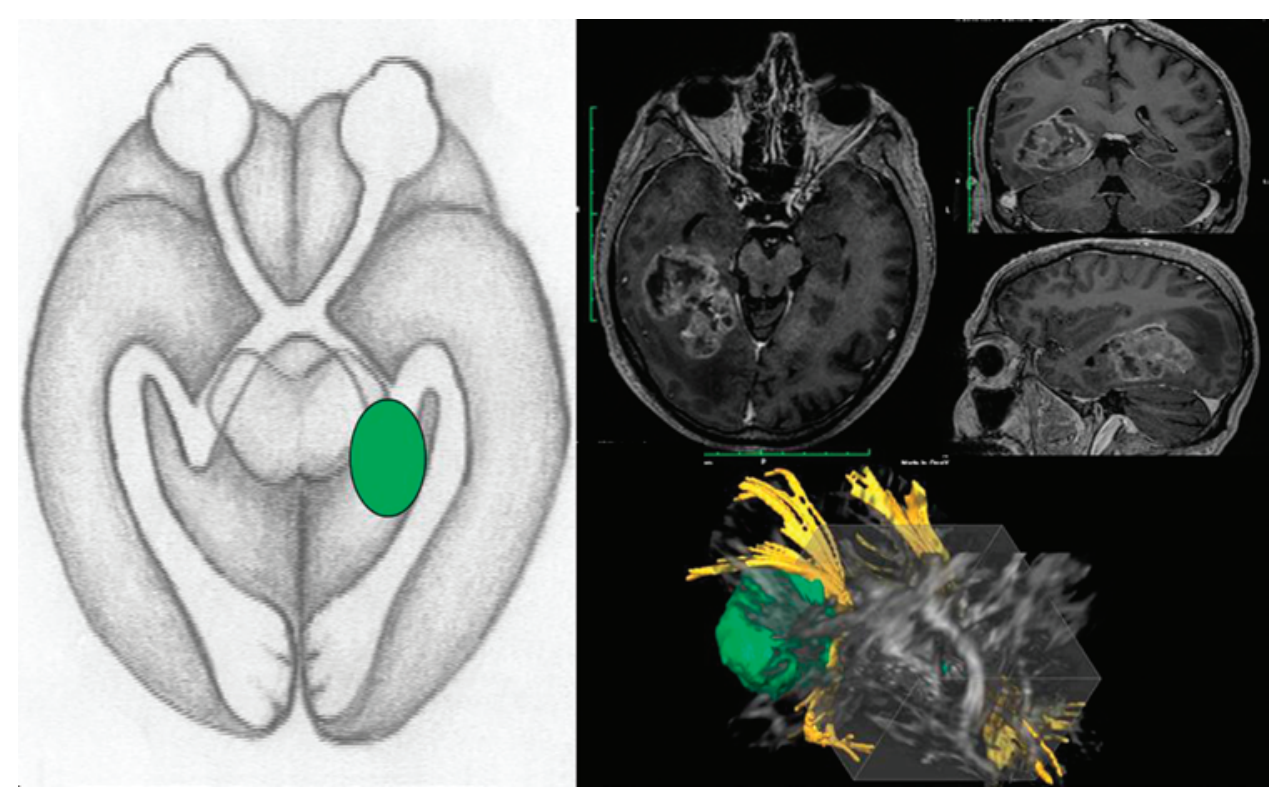

FIG. 7. Example of tumor in the dorsal temporomesial region. As depicted, fiber tracts of the OR (yellow) are shifted superiorly and laterally by the tumor (green). This tumor is difficult to access through an anterior transsylvian approach because of the long trajectory; however, it can be approached with maximum sparing of the OR by a supratentorial infraoccipital approach. Figure is available in color online only.

the MRI measurements and performed the operations in all 113 patients according to the recommended approaches. In 102 (90\%) patients, gross-total resection was accomplished. In 10 (9\%) patients the resection was subtotal, and in $1(1 \%)$ patient the tumor was only partially removed. General neurological (10 [9\%] of 113 patients) and surgical (9 [8\%] of 113 patients) morbidity rates were similar to previously published rates, ${ }^{7}$ as was the overall morbidity rate of less than $10 \%$ (Table 2).

To assess visual morbidity, we only used the cohort of 54 patients who had undergone CP by board-certified ophthalmologists (Table 3) because of a the higher inaccuracy of analysis and greater likelihood of missing small VFDs in the 59 patients who underwent finger perimetry
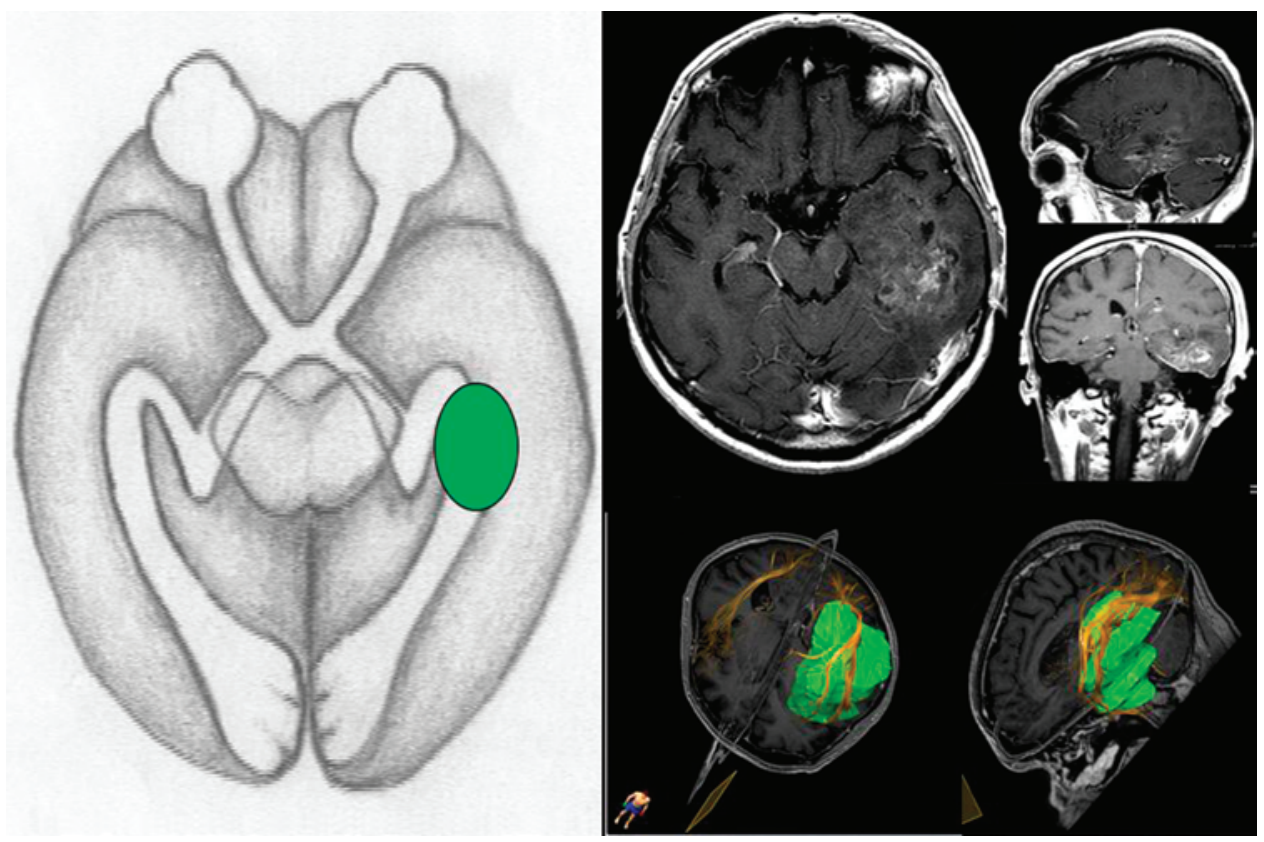

FIG. 8. Example of temporocentric tumor. As depicted, fiber tracts of the OR (yellow) are shifted laterally and superiorly by the tumor (green). Figure is available in color online only. 


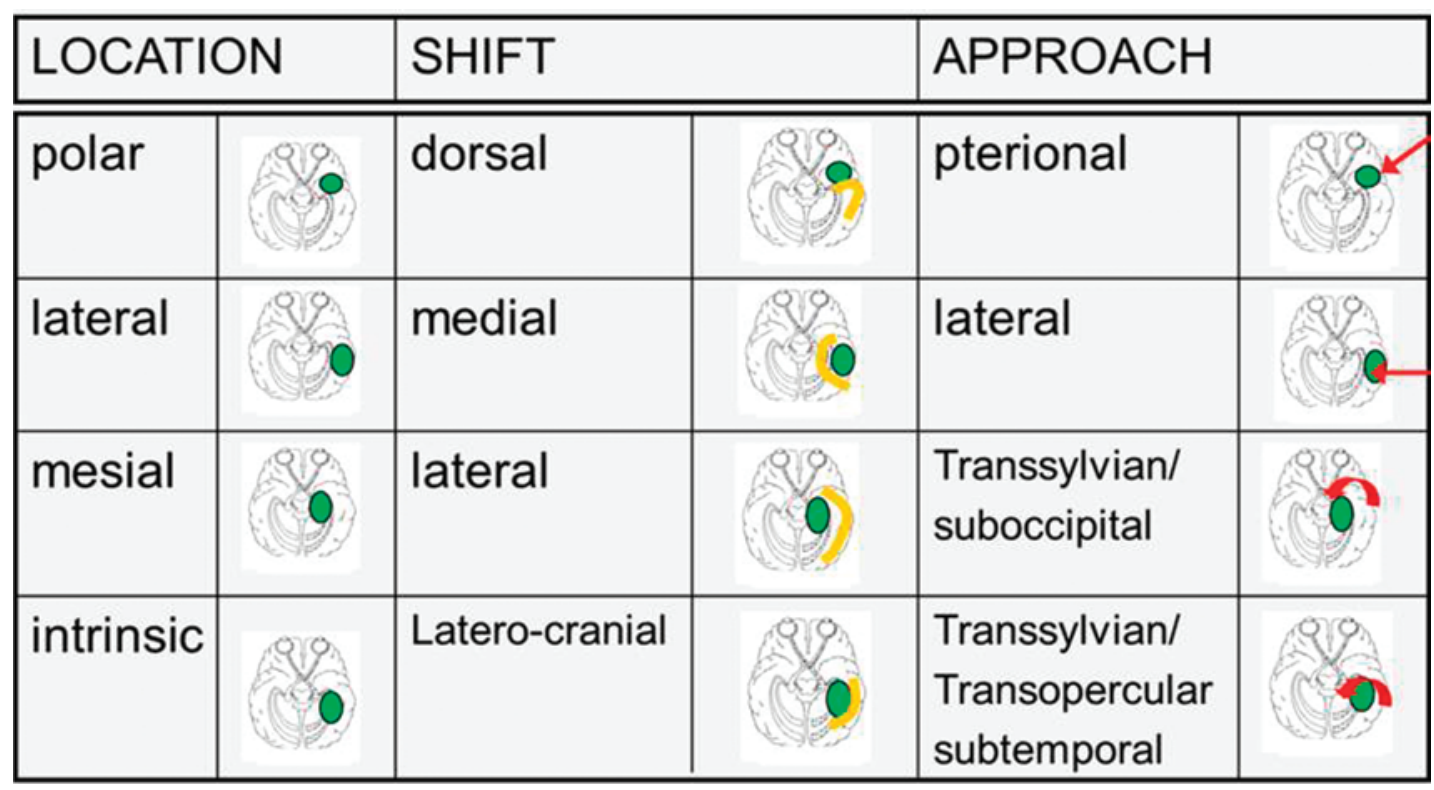

FIG. 9. Allocation of tumor locations within the temporal lobe to their predetermined directions of fiber shift and suggested surgical approaches aiming at maximum sparing of the OR. Figure is available in color online only.

only. (Note, however, that the incidence and distribution of VFDs in the finger perimetry group were comparable to those in the CP group [data not shown].) The overall incidence of new or worsened postoperatively impaired visual fields was $4 \%$. Among the $24 \%$ of patients with intact visual fields preoperatively, the incidence of new postoperative visual field morbidity was $8 \%$. Among the $76 \%$ of patients with existing visual field deficits preoperatively, a worsening of these deficits was encountered in $2 \%$, whereas $24 \%$ of the patients improved postoperatively (Fig. 10).

\section{Discussion}

Knowledge of the exact course of the OR in each patient with a temporal tumor may help improve neurosurgical results and avoid visual field deficits. The principal novel finding of our study is that tumors of the temporal lobe, whether gliomas or metastases, displace the OR rather than infiltrate and destroy it. Furthermore, we have

TABLE 2. Summary of temporary and permanent neurological morbidity in 10 patients as well as surgical morbidity in 9 patients

\begin{tabular}{cc}
\hline \multicolumn{1}{c}{ Morbidity } & No. of Cases \\
\hline Neurological ${ }^{*}$ & \\
\hline New VFD & $0 / 5$ \\
\hline Hemiparesis & $3 / 1$ \\
\hline Aphasia & $5 / 2$ \\
\hline Third nerve palsy & $4 / 2$ \\
\hline Surgical & \\
\hline Wound healing disorder & 7 \\
\hline Bleeding requiring revision & 2 \\
\hline
\end{tabular}

* Listed values represent temporary/permanent morbidities. identified reproducible patterns of OR fiber shift, which are dictated by the compartment of tumor origin within the temporal lobe. Thus, using our proposed classification system and the corresponding surgical approaches may help to significantly reduce visual field deficits in temporal lobe tumor surgery.

In the last 2 decades, tremendous insight into the anatomy of the OR was obtained through fiber dissection studies..$^{21,26}$ The LGB marks the beginning of the OR (Fig. $11 \mathrm{~A})$. In the LGB, as in the whole visual pathway, the optic fibers show a strict retinotopic arrangement. From the LGB onwards, the OR can be subdivided into 3 major fiber bundles: an anterior bundle, a central bundle, and an inferior bundle. The anterior bundle, which contains information from the contralateral upper visual field quadrant, extends anterior and superior to the temporal horn of the ventricle toward the temporal pole, where it forms the Meyer's loop, and then turns posterior to the primary visual cortex in the occipital lobe, below the calcarine sulcus. The central bundle contains visual information from the fovea centralis. Without forming a loop, it extends above the temporal horn straight to Brodmann area 19. The inferior bundle contains information from the contra-

TABLE 3. Summary of visual morbidity in 54 patients analyzed with both pre- and postoperative $\mathrm{CP}$

\begin{tabular}{|c|c|c|c|}
\hline \multicolumn{4}{|c|}{ No. (\%) } \\
\hline \multicolumn{2}{|c|}{ VFD Preop } & \multicolumn{2}{|c|}{ VFD Postop } \\
\hline No & $13(24)$ & No & $12(92)$ \\
\hline & & Yes & $1(8)$ \\
\hline \multirow[t]{3}{*}{ Yes } & $41(76)$ & Better & $10(24)$ \\
\hline & & Same & $30(73)$ \\
\hline & & Worse & $1(2)$ \\
\hline
\end{tabular}




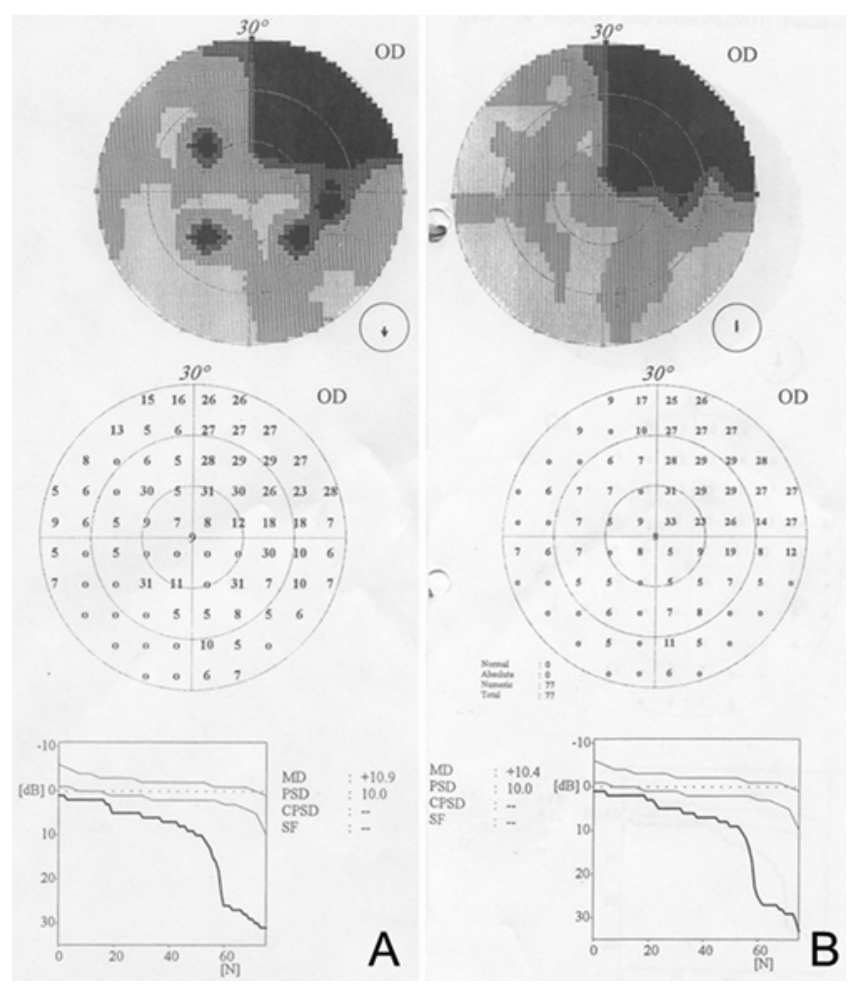

FIG. 10. Example of pre- and postoperative CP results for a patient with temporomesial tumor (same patient whose images appear in Fig. 6). A: Preoperative. B: Postoperative. As depicted, this patient suffered from a contralateral superior quadrantanopia. Postoperatively, no worsening of the VFD was documented. The patient was included in the group that was unaltered postoperatively. CPSD = corrected pattern standard deviation; $\mathrm{MD}=$ mean deviation; $\mathrm{OD}=$ oculus dexter; $\mathrm{PSD}=$ pattern standard deviation; SF = short-term fluctuation.

lateral lower quadrant. It extends lateral to the atrium toward an area above the calcarine sulcus (Fig. 11B). Given this knowledge, one can always predict the resulting VFD solely from the topographic location of a lesion within the temporal lobe. Notably, the visual tract spans an enormous distance, from the brain's most anterior to its most posterior end. To date, DTI is the only method capable of displaying cerebral white matter tracts in vivo. Over the last decade, the advances of DTI have revealed further significant insights into the fiber anatomy of the OR, 3,18,20,34 including a pronounced interindividual variability. ${ }^{3}$ These interindividual differences become even more relevant in situations in which tumors are growing within the temporal lobe.

Data in the present study demonstrate the feasibility of visualizing fibers of the visual pathway in tumor-bearing temporal lobes. To date, successful fiber tracking of the OR has been documented only in healthy subjects or in the context of epilepsy surgery. . $^{3,18,20,24}$ The possibility of a tumor causing adjacent anisotropy changes capable of impeding the visualization of white matter tracts (for example, by tumor edema, infiltration of fibers, or destruction of fibers) clearly needed to be considered. In the present study, we were able to qualitatively and quantitatively confirm the preoperative integrity of white matter tracts in all cases, even with invasive growing tumors. Moreover, we demonstrated distinct fiber displacement caused by temporal lobe tumors, suggesting that the fibers of the OR possess an impressive flexibility without loss of their integrity. There was no indication that fibers of the OR were substantially lost or destroyed even by invasively growing tumors. The assumption that preoperatively existing VFDs would be caused by the destructive nature of a tumor and were therefore irreversible may not apply. The realization that VFDs may, in fact, be improved by tumor removal (even after pharmacological minimization of tumor edema) may encourage neurosurgeons to invest greater effort, particularity in studying the tumor-fiber relations before any temporal surgery, and to take into account the individually altered fiber anatomy for their chosen approach. In our patient cohort, knowledge of the DTI data changed the surgical approach and helped to better define the surgical strategy. The improvement of preexisting symptoms or the absence of new or worsened symptoms in $95 \%$ of the cohort overall in the postoperative course confirms the value of the technique. Therefore, our results indicate a clear superiority in visual outcomes when the fiber tracking information is considered as compared with outcomes in previous studies in which fiber tracking had not been employed.

When analyzing the fiber tracking data in the 113 patients included in this study, patterns became evident, indicating that the direction of fiber shift is strictly predetermined by the anatomical location of the tumor. The observed direction of fiber displacement was always congruent with the origin of tumor growth. Lateral tumors, which extend medially, entrain the OR medially. Mesial tumors, expanding laterally, shift the fibers laterally. As shown in the coronal section of Fig. 11C, the origin of every temporomesial tumor lies medial to the OR. Thus, the positional relation between tumor and fiber tracts was always retained, even in space-occupying lesions. Polar tumors, which can only expand dorsally, push the OR correspondingly. Central tumors are always embedded within the most intricate architecture of the white matter tracts, which occupy the largest volume of the temporocentral region. 11,16,19,21,26,27,32 Besides the OR, this region includes the anterior commissure, the uncinate fasciculus, and the temporopontine and temporothalamic fibers, as well as many others, some of which are not yet fully described or understood. ${ }^{34}$ Those centric tumors tend to spread along the trajectories of the temporal stem they are incorporated into. Our data suggest that those tumors push the OR laterally and superiorly. We assume that large temporocentric lesions, which grow into the frontal lobe, may therefore pass through the temporal stem medial to the OR. Tumors of the medial occipitotemporal (fusiform) gyrus naturally push the OR cranially.

Today, routine tracking of the OR before every temporal surgery is not (yet) applied by every neurosurgeon for various reasons. Moreover, not every MRI facility offers DTI imaging. And there are patients with temporal tumors (for example, those with pacemakers) who simply cannot undergo DTI processing as part of their preoperative workup. In these cases, knowledge of the fixed allocation of tumor location and fiber shift may prove useful. To minimize injury to the OR, safe approaches can be chosen according to those outlined in Fig. 9. 

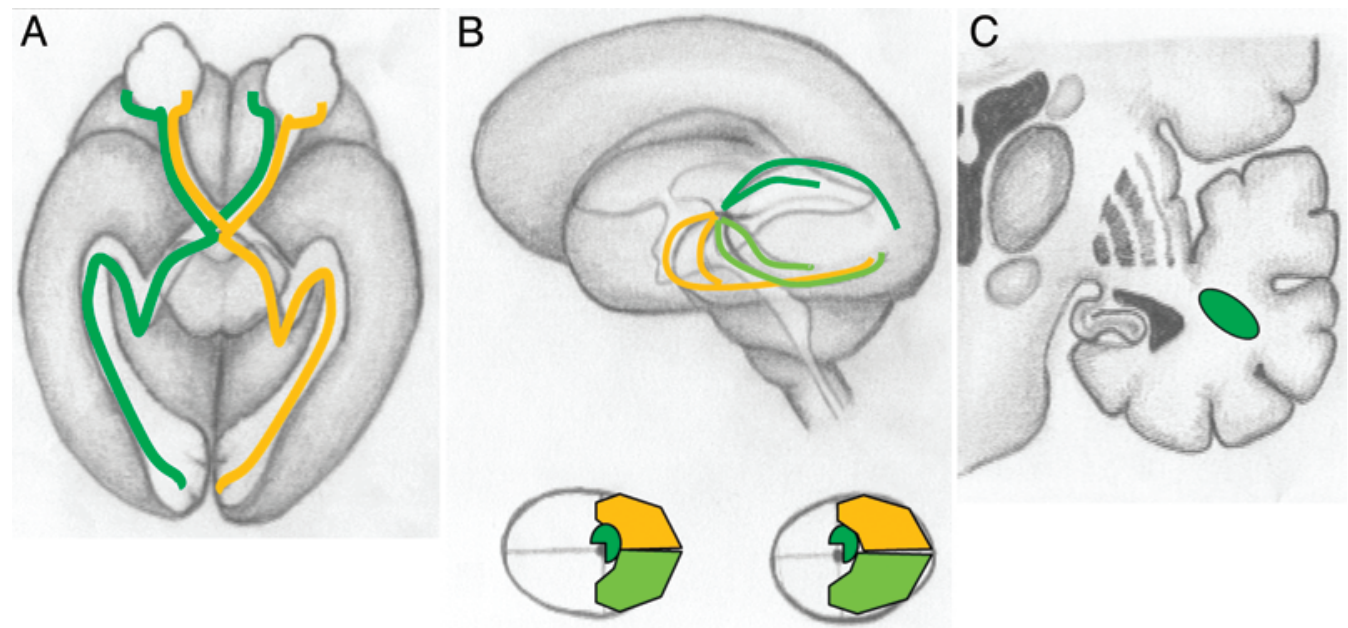

FIG. 11. Diagram (A) of the optic pathway. The OR extends from the LGB to the calcarine cortex. From the LGB onward (B), the OR is divided into 3 bundles, each representing a specific area of the visual field: an anterior bundle, containing information from the contralateral upper quadrant (yellow); an inferior bundle, containing information from the contralateral lower quadrant (light green); and a central bundle, containing information from the central visual field (dark green). As discernible on coronal sections (C), the OR is located lateral (and slightly superior) to the temporomesial structures. Figure is available in color online only.

Previously, we proposed and applied the division of temporal lobe tumors into 4 basic topographic types.? The division was based on the graded assembly of the temporal lobe from outer over intermediate to inner layers on axial sections, and thus reflected the ascending ontogenetic ages of the lobe from outer to inner layers. In that earlier study, we also demonstrated that a strict allocation of anatomical tumor location with a surgical approach - that is, lateral and polar tumor location with transcortical approaches and limbic and central/intrinsic tumor locations with transsylvian approaches-resulted in minimal neurological morbidity other than VFDs, including hemiparesis and aphasia, as well as minimal vascular complication and good gross-total resection. ${ }^{7}$ Therefore, we conclude that the implementation of the proposed surgical approaches (Fig. 9) leads to good postoperative results in general, beyond the reduction of VFDs.

Our previous classification of approaches ${ }^{7}$ was rather intuitive. We found that the applied approaches resulted in a maximum reduction of visual field deficits. After executing the tractography, it became evident that the direction of fiber shifts follows rather rigid patterns that are dependent solely on the anatomical tumor location. Therefore, one can ultimately predict the resulting shift of the OR merely based on the topographic location of a lesion, even without exact knowledge of the DTI data. In fact, one may dispense with DTI fiber tracking to reduce visual morbidity in temporal lobe surgery if one adheres to the classification of approaches outlined in Fig. 9.

\section{Conclusions}

Preoperative DTI fiber tracking of the OR may be a valuable tool for planning approaches to temporal lobe tumors. Its use may lead to a reduction in postoperative visual morbidity. Temporal lobe tumors lead to a dislocation of the OR rather than its destruction. The direction of fiber shift depends exclusively on the tumor topography and is therefore predictable. Consequently, the above-proposed allocation of surgical approaches to tumor topography may prove helpful and improve outcomes if DTI is not feasible.

\section{References}

1. Bammer R, Acar B, Moseley ME: In vivo MR tractography using diffusion imaging. Eur J Radiol 45:223-234, 2003

2. Basser PJ, Pajevic S, Pierpaoli C, Duda J, Aldroubi A: In vivo fiber tractography using DT-MRI data. Magn Reson Med 44:625-632, 2000

3. Chen X, Weigel D, Ganslandt O, Buchfelder M, Nimsky C: Prediction of visual field deficits by diffusion tensor imaging in temporal lobe epilepsy surgery. Neuroimage 45:286-297, 2009

4. Ciccarelli O, Toosy AT, Hickman SJ, Parker GJ, WheelerKingshott CA, Miller DH, et al: Optic radiation changes after optic neuritis detected by tractography-based group mapping. Hum Brain Mapp 25:308-316, 2005

5. Conturo TE, Lori NF, Cull TS, Akbudak E, Snyder AZ, Shimony JS, et al: Tracking neuronal fiber pathways in the living human brain. Proc Natl Acad Sci U S A 96:10422-10427, 1999

6. Ebeling U, Reulen HJ: Neurosurgical topography of the optic radiation in the temporal lobe. Acta Neurochir (Wien) 92:29-36, 1988

7. Faust K, Schmiedek P, Vajkoczy P: Approaches to temporal lobe lesions: a proposal for classification. Acta Neurochir (Wien) 156:409-413, 2014

8. Hofer S, Karaus A, Frahm J: Reconstruction and dissection of the entire human visual pathway using diffusion tensor MRI. Front Neuroanat 4:15, 2010

9. Horel JA, Misantone LJ: Visual discrimination impaired by cutting temporal lobe connections. Science 193:336-338, 1976

10. Hughes TS, Abou-Khalil B, Lavin PJ, Fakhoury T, Blumenkopf B, Donahue SP: Visual field defects after temporal lobe resection: a prospective quantitative analysis. Neurology 53:167-172, 1999

11. Jellison BJ, Field AS, Medow J, Lazar M, Salamat MS, Alexander AL: Diffusion tensor imaging of cerebral white matter: 
a pictorial review of physics, fiber tract anatomy, and tumor imaging patterns. AJNR Am J Neuroradiol 25:356-369, 2004

12. Krolak-Salmon P, Guenot M, Tiliket C, Isnard J, Sindou M, Mauguiere F, et al: Anatomy of optic nerve radiations as assessed by static perimetry and MRI after tailored temporal lobectomy. Br J Ophthalmol 84:884-889, 2000

13. McDonald CR, Hagler DJ Jr, Girard HM, Pung C, Ahmadi ME, Holland D, et al: Changes in fiber tract integrity and visual fields after anterior temporal lobectomy. Neurology 75:1631-1638, 2010

14. Mori S, van Zijl PC: Fiber tracking: principles and strategies - a technical review. NMR Biomed 15:468-480, 2002

15. Peltier J, Travers N, Destrieux C, Velut S: Optic radiations: a microsurgical anatomical study. J Neurosurg 105:294-300, 2006

16. Peltier J, Verclytte S, Delmaire C, Pruvo JP, Godefroy O, Le Gars D: Microsurgical anatomy of the temporal stem: clinical relevance and correlations with diffusion tensor imaging fiber tracking. J Neurosurg 112:1033-1038, 2010

17. Pierpaoli C, Jezzard P, Basser PJ, Barnett A, Di Chiro G: Diffusion tensor MR imaging of the human brain. Radiology 201:637-648, 1996

18. Powell HWR, Parker GJM, Alexander DC, Symms MR, Boulby PA, Wheeler-Kingshott CAM, et al: MR tractography predicts visual field defects following temporal lobe resection. Neurology 65:596-599, 2005

19. Rubino PA, Rhoton AL Jr, Tong X: Oliveira Ed: Three-dimensional relationships of the optic radiation. Neurosurgery 57: $219-227,2005$

20. Sherbondy AJ, Dougherty RF, Napel S, Wandell BA: Identifying the human optic radiation using diffusion imaging and fiber tractography. J Vis 8:12.1-12.11, 2008

21. Sincoff EH, Tan Y, Abdulrauf SI: White matter fiber dissection of the optic radiations of the temporal lobe and implications for surgical approaches to the temporal horn. J Neurosurg 101:739-746, 2004

22. Staempfli P, Rienmueller A, Reischauer C, Valavanis A, Boesiger P, Kollias S: Reconstruction of the human visual system based on DTI fiber tracking. J Magn Reson Imaging 26:886-893, 2007

23. Tao XF, Wang ZQ, Gong WQ, Jiang QJ, Shi ZR: A new study on diffusion tensor imaging of the whole visual pathway fiber bundle and clinical application. Chin Med J (Engl) 122:178-182, 2009

24. Taoka T, Sakamoto M, Nakagawa H, Nakase H, Iwasaki S, Takayama K, et al: Diffusion tensor tractography of the Meyer loop in cases of temporal lobe resection for temporal lobe epilepsy: correlation between postsurgical visual field defect and anterior limit of Meyer loop on tractography. AJNR Am J Neuroradiol 29:1329-1334, 2008

25. Tournier JD, Calamante F, King MD, Gadian DG, Connelly A: Limitations and requirements of diffusion tensor fiber tracking: an assessment using simulations. Magn Reson Med 47:701-708, 2002

26. Türe U, Yaşargil MG, Friedman AH, Al-Mefty O: Fiber dissection technique: lateral aspect of the brain. Neurosurgery 47:417-427, 2000

27. Türe U, Yaşargil MG, Pait TG: Is there a superior occipitofrontal fasciculus? A microsurgical anatomic study. Neurosurgery 40:1226-1232, 1997

28. Van Buren JM, Baldwin M: The architecture of the optic radiation in the temporal lobe of man. Brain 81:15-40, 1958

29. Winston GP, Daga P, Stretton J, Modat M, Symms MR, McEvoy AW, et al: Optic radiation tractography and vision in anterior temporal lobe resection. Ann Neurol 71:334-341, 2012

30. Yam D, Nicolle D, Steven DA, Lee D, Hess T, Burneo JG: Visual field deficits following anterior temporal lobectomy: long-term follow-up and prognostic implications. Epilepsia 51:1018-1023, 2010

31. Yamamoto A, Miki Y, Urayama S, Fushimi Y, Okada T, Hanakawa T, et al: Diffusion tensor fiber tractography of the optic radiation: analysis with 6-, 12-, 40-, and 81-directional motion-probing gradients, a preliminary study. AJNR Am J Neuroradiol 28:92-96, 2007

32. Yaşargil MG, Türe U, Yaşargil DC: Impact of temporal lobe surgery. J Neurosurg 101:725-738, 2004

33. Yogarajah M, Focke NK, Bonelli S, Cercignani M, Acheson J, Parker GJ, et al: Defining Meyer's loop-temporal lobe resections, visual field deficits and diffusion tensor tractography. Brain 132:1656-1668, 2009

34. Zhang Y, Wan SH, Wu GJ, Zhang XL: Magnetic resonance diffusion tensor imaging and diffusion tensor tractography of human visual pathway. Int J Ophthalmol 5:452-458, 2012

\section{Disclosure}

The authors report no conflict of interest concerning the materials or methods used in this study or the findings specified in this paper.

\section{Author Contributions}

Conception and design: Faust. Acquisition of data: Faust. Analysis and interpretation of data: Faust. Drafting the article: Faust. Critically revising the article: Vajkoczy. Reviewed submitted version of manuscript: Faust. Approved the final version of the manuscript on behalf of both authors: Vajkoczy. Statistical analysis: Faust. Administrative/technical/material support: Faust. Study supervision: Vajkoczy.

\section{Correspondence}

Peter Vajkoczy, Department of Neurosurgery, Charité Universitätsmedizin Berlin, Augustenburger Platz 1, Berlin 13353, Germany.email: peter.vajkoczy@charite.de. 